\title{
Pacific
}

Journal of

Mathematics

\section{ON THE UNIFORM APPROXIMATION PROBLEM FOR THE SQUARE OF THE CAUCHY-RIEMANN OPERATOR}

\author{
JoAn MANuel Verdera MELEnCHÓN
}




\section{ON THE UNIFORM APPROXIMATION PROBLEM FOR THE SQUARE OF THE CAUCHY-RIEMANN OPERATOR}

\section{JOAN VERDERA}

Let $X$ be a compact subset of the plane and $f$ a continuous function on $X$ satisfying the equation $\bar{\partial}^{2} f=0$ in the interior of $X$. It is unknown whether $f$ can be uniformly approximated on $X$ by functions $g$ satisfying the equation $\bar{\partial}^{2} g=0$ in some neighbourhood (depending on $g$ ) of $X$. We show that this is the case under the additional assumption that $f$ satisfies a Dini-type continuity condition.

1. Introduction. Let $L$ be a constant coefficients elliptic differential operator in $\mathbb{R}^{d}$. Given a compact $X \subset \mathbb{R}^{d}$ let $H(X, L)$ be the closure in $C(X)$ of the set

$$
\left\{\left.f\right|_{X}: L f=0 \text { on some neighbourhood of } X\right\} .
$$

It is clear that a function in $H(X, L)$ necessarily belongs to

$$
h(X, L)=C(X) \cap\{L f=0 \text { on the interior of } X\} .
$$

The uniform approximation problem for the operator $L$ consists in characterizing those $X$ for which $H(X, L)=h(X, L)$. Since $h(X, L)=C(X)$ if and only if $X$ is nowhere dense, our problem restricted to nowhere dense compact sets becomes that of describing those $X$ for which $H(X, L)=C(X)$.

A complete solution for $L=\Delta$ (the Laplacian) was independently obtained in the forties by Deny [2] and Keldysh [6] using a duality approach relying on potential theoretic methods. Denoting by Cap the Wiener capacity of classical potential theory, their result can be stated as follows.

THEOREM (Deny-Keldysh). The identity $H(X, \Delta)=h(X, \Delta)$ occurs if and only if one has $\operatorname{Cap}(B \backslash \stackrel{\circ}{X})=\operatorname{Cap}(B \backslash X)$ for each open ball $B$.

Vitushkin [11] solved in the sixties the problem for $L=\bar{\partial}$ (the Cauchy-Riemann operator in the plane) introducing his far reaching 
constructive scheme for the approximation, based on a localization procedure and the so called matching coefficients technique.

Let $\alpha$ stand for continuous analytic capacity (see [3] or [11]). We then have

THEOREM (Vitushkin). The identity $H(X, \bar{\partial})=h(X, \bar{\partial})$ occurs if and only if one has $\alpha(\Delta \backslash \stackrel{\circ}{X})=\alpha(\Delta \backslash X)$ for each open disc $\Delta$.

In spite of the formal analogy between the above two statements, no unified proof of them has been found yet. This fact helps to explain why very little is known for other operators, in particular for $\Delta^{n}$, $n>1, d \geq 2$ or for $\bar{\partial}^{n}, n>1$, in the plane.

In this paper the special case $d=2$ and $L=\bar{\partial}^{2}$ is considered. Although we have not been able to solve the problem we do prove a result which seems to be quite close to the most plausible conjecture one is able to formulate.

Theorem. Let $X \subset \mathbb{C}$ be compact. Then each Dini-continuous function in $h\left(X, \bar{\partial}^{2}\right)$ is in $H\left(X, \bar{\partial}^{2}\right)$.

Conjecture. For each compact $X \subset \mathbb{C}$ one has

$$
H\left(X, \bar{\partial}^{2}\right)=h\left(X, \bar{\partial}^{2}\right) \text {. }
$$

To understand the conjecture one must realize that the capacitary conditions in the Deny-Keldysh and Vitushkin theorems arise because the fundamental solutions of the involved operators are unbounded functions. This is not the case for the fundamental solution $\frac{1}{\pi} \frac{\bar{z}}{z}$ of $\bar{\partial}^{2}$ and so we get a first reason to rule out the existence of capacitary conditions on $X$ necessary for (1). But it has been pointed out [7] that one should also take into account the capacities associated to continuous functions and the kernels given by the first partial derivatives of the fundamental solution, for example, $1 / z=\bar{\partial}(\bar{z} / z)$ and $\bar{z} / z^{2}=-\partial(\bar{z} / z)$. One can easily check that these capacities vanish on lines and thus no examples like those constructed by Hedberg [5] in connection with the $L^{p}$ approximation problem for $\bar{\partial}^{2}, 2 \leq p<\infty$, can be used to disprove the conjecture (see $\S 6$ ).

We list now some particular instances in which (1) has been verified:

1. $X$ is nowhere dense [10].

2. The complement of $X$ has finitely many connected components [1].

3. The inner boundary of $X$ is countable [12]. 
The interested reader is urged to consult [7] in which a formal ana$\log$ of the problem considered here is solved. In fact the scheme of the proof of our theorem is parallel to that of [7]: there is a first constructive part exploiting a covering lemma due to Mateu and then a duality argument in which the differentiability properties of certain potentials play an important role. The Dini type condition allows us to surmount the difficulties related to the bad behaviour of classical operators (more concretely Cauchy and Beurling transforms) under the supremum norm.

In $\S 2$ we state some known results and establish some notation to be used later. Section 3 is devoted to the proof of a technical lemma. In $\S 4$ we show how to approximate some Cauchy potentials of measures. The proof of the theorem is presented in $\S 5$. To support the conjecture we prove in $\S 6$ that (1) holds for the string of beads.

2. Background notation and results. In this section we establish some notation and we recall some known facts to be used throughout the rest of the paper.

A. Newtonian capacity. Newtonian capacity is the set function defined on Borel sets $E \subset \mathbb{C}$ by

$$
C(E)=\sup \|\mu\|
$$

where the supremum is taken over all positive measures $\mu$ with compact support contained in $E$ and satisfying $\frac{1}{|z|} * \mu \leq 1$ on $E$ (or, equivalently, on $\mathbb{C}$ ). In fact, one can besides require the continuity of $\frac{1}{|z|} * \mu$ without altering the supremum in (2).

There is a dual expression for newtonian capacity, namely

$$
C(E)=\inf \|\lambda\|
$$

the infimum being over all positive measures $\lambda$ such that $\frac{1}{|z|} * \lambda \geq 1$ on $E$. From that it readily follows that $\frac{1}{|z|} * \lambda$ is finite $C$-almost everywhere for all complex Borel measures $\lambda$.

One can easily get a lower bound for $C(E)$ in terms of Hausdorff content. Let $h(t), t>0$, be a measure function (that is, nondecreasing and continuous) of the form $h(t)=w(t) t$ with

$$
\int \frac{w(t)}{t} d t<\infty \text {. }
$$

Set

$$
\varepsilon(\delta)=w(\delta)+\int_{0}^{\delta} \frac{w(t)}{t} d t, \quad \delta>0
$$


Then

$$
M^{h}(E) \leq \varepsilon(d) C(E)
$$

where $d$ is the diameter of $E$ and $M^{h}(E)=\inf \sum_{j} h\left(d_{j}\right)$, the infimum being taken over all coverings of $E$ by squares of diameter $d_{j}$.

We refer the reader to [4] for a convenient account on newtonian capacity.

B. A covering lemma. A family of discs $\left(\Delta_{j}\right)$ is said to be almost disjoint with constant $N$ provided each point in $\mathbb{C}$ belongs to at most $N$ of the discs $\Delta_{j}$.

Given a set $E$ and a disc $\Delta$ with radius $\delta$ we say that $\Delta$ has the three points property (with constant $\eta, 0<\eta<1$ ) with respect to $E$ if we can find points $z_{1}, z_{2}$ and $z_{3}$ in $\Delta \cap E$ such that $\left|z_{1}-z_{2}\right| \geq$ $\eta \delta$ and $d\left(z_{3}, l\left(z_{1}, z_{2}\right)\right) \geq \eta \delta$, where $l\left(z_{1}, z_{2}\right)$ is the straight line through $z_{1}$ and $z_{2}$.

The relevance of this notion in this paper is due to the fact that it allows us to produce a function annihilated by $\bar{\partial}^{2}$ off a disc and with a given expansion at $\infty$ up to order 2 . More concretely we have

2.1. Lemma. Let $\delta>0$ and $0<\eta<1$. Let $z_{1}, z_{2}, z_{3}$ be three points satisfying, for some positive constant $C$,

$$
\left|z_{j}-z_{k}\right| \leq C \delta, \quad j, k=1,2,3,
$$

and

$$
\min \left\{\left|z_{1}-z_{2}\right|, d\left(z_{3}, l\left(z_{1}, z_{2}\right)\right)\right\} \geq \eta \delta .
$$

Assume $a_{0}, a_{1}, b_{1} \in \mathbb{C}$ and

$$
\left|a_{0}\right| \leq C w, \quad\left|a_{1}\right|,\left|b_{1}\right| \leq C \delta w,
$$

for some $w>0$. Then there exists a function $g$ satisfying $\bar{\partial}^{2} g=0$ on $\mathbb{C} \backslash\left\{z_{1}, z_{2}, z_{3}\right\}$,

$$
g(z)=a_{0} \frac{\bar{z}}{z}+a_{1} \frac{1}{z} b_{1} \frac{\bar{z}}{z^{2}}+0\left(|z|^{-2}\right), \quad \text { as } z \rightarrow \infty,
$$

and

$$
\|g\|_{\infty} \leq C \eta^{-2} w
$$

REMARK. In our application of the above statement, $w$ will be $w(\delta)$, the modulus of continuity of the function to be approximated, and $C$ and $\eta$ constants depending only on $w(\delta)$. 
Proof of 2.1. Set $g=\frac{\bar{z}}{z} * \mu$, where $\mu=\lambda_{1} \delta_{z_{1}}+\lambda_{2} \delta_{z_{2}}+\lambda_{3} \delta_{z_{3}}$ and the coefficients $\lambda_{j}$ are chosen so that $g$ has the required expansion at $\infty$.

2.2. Lemma (Mateu [7]). Let $h(t)=t w(t)$ be a measure function with $w$ nondecreasing and satisfying $w(2 t) \leq C w(t)$. Then for any compact $K \subset \mathbb{C}$ there exist a finite family of discs $\left(\Delta_{j}\right)$ which can be divided into two subfamilies $\left(\Delta_{j}^{g}\right)$ and $\left(\Delta_{j}^{b}\right)$ (the superscripts $g$ and $b$ stand for good and bad) in such a way that the following holds (with constants depending on $w$ but not on $K$ ).

(a) $K \subset \bigcup_{j} \Delta_{j}$.

(b) For some $\lambda=\lambda(w)>1,\left(\lambda \Delta_{j}\right)$ is almost disjoint (with constant depending only on $w$ ).

(c) Each $\Delta_{j}^{g}$ has the three points property (with constant depending only on $w$ ) with respect to $K$.

(d) $\sum_{j} h\left(\delta_{j}^{b}\right) \leq C M^{h}(K)$, where $\delta_{j}^{b}$ denotes the radius of the disc $\Delta_{j}^{b}$.

(e) For each disc $\Delta$ of radius $\delta$

$$
\sum_{\Delta_{j}^{b} \subset \Delta} h\left(\delta_{j}^{b}\right) \leq C h(\delta) .
$$

REMARKs. 1. It is not difficult to realize that moreover one has

$$
\sum_{j} w\left(\delta_{j}\right) \delta_{j}^{2} \leq C \delta M^{h}(K) .
$$

2. It has been recently shown in [8] that is not possible, for $h(t)=t$, to construct a family $\left(\Delta_{j}\right)$ satisfying $(\mathrm{a}),(\mathrm{b})$ and $(\mathrm{d})$ (with $\Delta_{j}^{b}$ replaced by $\Delta_{j}$ ).

3. A lemma. In this section we give a proof of the following technical lemma.

3.1. Lemma. Let $w(t), t>0$, be a non-decreasing continuous function satisfying $w(2 t) \leq C w(t)$ and $\int_{0}^{1} \frac{w(t)}{t} d t<\infty$. Suppose that $\left(\Delta_{j}\right)$ is a finite family of discs with centers $z_{j}$ and radii $\delta_{j}$ such that $\left(\lambda \Delta_{j}\right)$ is almost disjoint for some $\lambda>1$. Then the following holds.

(i) For some constant $C>0$

$$
\sum_{j} \min \left(w\left(\delta_{j}\right), \frac{w\left(\delta_{j}\right) \delta_{j}^{2}}{\left|z-z_{j}\right|^{2}}\right) \leq C \varepsilon(d), \quad z \in \mathbb{C},
$$


where $d$ is the diameter of $\bigcup_{j} \Delta_{j}$ and

$$
\varepsilon(\delta)=w(\delta)+\int_{0}^{\delta} \frac{w(t)}{t} d t, \quad \delta>0
$$

(ii) If moreover the family $\left(\Delta_{j}\right)$ satisfies the packing condition

$$
\sum_{\Delta_{J} \subset \Delta} w\left(\delta_{j}\right) \delta_{j} \leq C w(\delta) \delta
$$

for each disc $\Delta$ of radius $\delta$, then

$$
\sum_{j} \min \left(w\left(\delta_{j}\right), \frac{w\left(\delta_{j}\right) \delta_{j}}{\left|z-z_{j}\right|}\right) \leq C \varepsilon(\delta), \quad z \in \mathbb{C} .
$$

Proof. To prove (3) (and (5)) it is enough to assume $z \in 2 D, D$ being a disc of radius $d$ containing $\bigcup_{j} \Delta_{j}$. In fact if $z \notin 2 D$ then

$$
\sum_{j} \min \left(w\left(\delta_{j}\right), \frac{w\left(\delta_{j}\right) \delta_{j}^{2}}{\left|z-z_{j}\right|^{2}}\right) \leq \sum_{j} \frac{w\left(\delta_{j}\right) \delta_{j}^{2}}{\left|z-z_{j}\right|^{2}} \leq \frac{w(d)}{d^{2}} \sum_{j} \delta_{j}^{2} \leq C w(d)
$$

where in the last inequality almost disjointness was used.

We argue similarly for (5) applying the packing condition to the test disc $D$.

Fix then $z \in 2 D$. Set $\left.J=\left\{j: z \notin \lambda \Delta_{j}\right]\right\}$ and $J^{*}=\left\{j: z \in \lambda \Delta_{j}\right\}$. Thus

$$
\sum_{j \in J^{*}} \min \left(w\left(\delta_{j}\right), \frac{w\left(\delta_{j}\right) \delta_{j}^{2}}{\left|z-z_{j}\right|^{2}}\right) \leq \# J^{*} w(d) .
$$

To estimate the above sum over the indices $j \in J$ we proceed as follows. Set $\mu_{j}=w\left(\delta_{j}\right) \chi_{j}(\zeta) d x d y, j \in J$, where $\chi_{j}$ stands for the characteristic function of $\Delta_{j}$. Hence

$$
\frac{w\left(\delta_{j}\right) \delta_{j}^{2}}{\left|z-z_{j}\right|^{2}} \leq C \int \frac{d \mu_{j}(\zeta)}{|z-\zeta|^{2}}
$$

and so

$$
\sum_{j \in J} \min \left(w\left(\delta_{j}\right), \frac{w\left(\delta_{j}\right) \delta_{j}^{2}}{\left|z-z_{j}\right|^{2}}\right) \leq C \int \frac{d \mu(\zeta)}{|z-\zeta|^{2}},
$$

where $\mu=\sum_{j \in J} \mu_{j}$. Write $\mu(r)=\mu(\Delta(z, r))$. Then $\mu(r)=0$ provided $r$ is small enough, because $z$ does not belong to the support of $\mu$. Consequently one has

$$
\int \frac{d \mu(\zeta)}{|z-\zeta|^{2}}=\int_{0}^{3 d} \frac{d \mu(r)}{r^{2}}=\frac{\mu(3 d)}{(3 d)^{2}}+2 \int_{0}^{3 d} \frac{\mu(r)}{r^{3}} d r .
$$


Now it is clearly sufficient to show that

$$
\mu(r) \leq C w(r) r^{2}, \quad r>0 .
$$

To get (6) one writes

$$
\begin{aligned}
\mu(r) & =\sum_{j \in J} \mu_{j}\left(\Delta_{j} \cap \Delta(z, r)\right) \\
& \leq \sum_{\delta_{j} \leq r} w\left(\delta_{j}\right) \pi \delta_{j}^{2}+\sum_{\delta_{j}>r} w\left(\delta_{j}\right) \pi r^{2} \equiv \mathrm{I}+\mathrm{II},
\end{aligned}
$$

where only indices $j \in J$ with $\Delta_{j} \cap \Delta(z, r) \neq \varnothing$ are considered in the above sums.

If $\delta_{j} \leq r$ then $\Delta_{j} \subset \Delta(z, 3 r)$ and so

$$
\mathrm{I} \leq \sum_{\Delta_{J} \subset \Delta(z, 3 r)} w\left(\delta_{j}\right) \delta_{j}^{2} \leq C w(r) r^{2} .
$$

If $\delta_{j}>r$ then $r \geq(\lambda-1) \delta_{j}$ and so $\mathrm{II} \leq C w(r) r^{2} N$, where $N$ is the number of indices $j \in J$ with $\delta_{j}>r$ and $\Delta_{j} \cap \Delta(z, r) \neq \varnothing$. It turns out that $N$ can be estimated by some constant, because

$$
\begin{aligned}
4 \pi r & =\text { length } \partial \Delta(z, 2 r) \geq \text { length } \bigcup_{j}\left(\Delta_{j} \cap \partial \Delta(z, 2 r)\right) \\
& \geq C \sum_{j} \text { length } \Delta_{j} \cap \partial \Delta(z, 2 r) \geq C r N .
\end{aligned}
$$

This completes the proof of (i).

The proof of (ii) is essentially the same. One uses the packing assumption in estimating the analog of the term I above.

4. Approximation of some Cauchy transforms. This section is devoted to the proof of the following

4.1. Theorem. Let $X \subset \mathbb{C}$ be compact and let $\mu$ be a complex Borel measure whose support does not intersect the interior of $X$ and such that $(1 /|z|) *|\mu|$ is continuous on $\mathbb{C}$. Then if $P=(1 / z) * \mu$ and $Q=\left(\bar{z} / z^{2}\right) * \mu$ we have that

$$
P, Q, P^{2} \text { and } P Q \in H\left(X, \bar{\partial}^{2}\right) \text {. }
$$

REMARK. The function $P$ is continuous on $X$ and analytic on $\stackrel{\circ}{X}$. Although its modulus of continuity may vanish at zero as slowly as we wish, some Dini type condition is implicit in the fact that $(1 /|z|) *|\mu|$ 
is continuous. It is not known whether a function $f$, continuous on $X$ and analytic on $\stackrel{\circ}{X}$, belongs to $H\left(X, \bar{\partial}^{2}\right)$, even under the additional assumption that $f$ is the Cauchy transform of some measure.

The proof of 4.1 goes by duality. Let $\lambda$ be a complex Borel measure on $X$ annihilating $H\left(X, \bar{\partial}^{2}\right)$. Then the potential $F=(\bar{z} / z) * \lambda$ vanishes on the complement of $X$ and, on the other hand, we have

$$
\int P(z) d \lambda(z)=-\int\left(\frac{1}{z} * \lambda\right) d \mu=-\int \bar{\partial} F(z) d \mu(z) .
$$

It can be shown that $F$ is continuous except at those points where $\lambda$ has a positive mass [10]. This implies that $F$ vanishes on $(\stackrel{\circ}{X})^{c}$ except, eventually, on a countable set.

Therefore, we are left with the task of proving that $\bar{\partial} F$ vanishes on $(\stackrel{\circ}{X})^{c} \quad \mu$-almost everywhere and this will be achieved by studying ordinary differentiability properties of $F$.

4.2. Lemma. Let $\lambda$ be a complex Borel measure and set $F=$ $(\bar{z} / z) * \lambda$. Then $F$ is differentiable in the ordinary sense except on a set of newtonian capacity zero.

Proof. We have, in the distributions sense, $\bar{\partial} F=(1 / z) * \lambda$ and $\partial F=-\left(\bar{z} / z^{2}\right) * \lambda$. Assume, without loss of generality, that $\lambda$ is positive and let $a$ be a point with $|z|^{-1} * \lambda(a)<\infty$. We are going to show that

$$
|F(z)-F(a)-\alpha(z-a)-\beta(\bar{z}-\bar{a})|=o(|z|), \quad \text { as } z \rightarrow a,
$$

where $\alpha=\left(-\left(\bar{z} / z^{2}\right) * \lambda\right)(a)$ and $\beta=((1 / z) * \lambda)(a)$.

To prove (7) we assume $a=0$ and according to $[9$, p. 244] we write

$$
\mid F(z)-F(0)-(\alpha z+\beta \bar{z} \mid \leq \mathrm{I}+\mathrm{II}+\mathrm{III},
$$

where

$$
\begin{aligned}
& \mathrm{I}=\left|\int_{|\zeta|>2|z|}\left(\frac{\bar{z}-\bar{\zeta}}{z-\zeta}-\frac{\bar{\zeta}}{\zeta}\right) d \lambda(\zeta)-(\alpha z+\beta z)\right|, \\
& \mathrm{II}=\left|\int_{|\zeta| \leq 2|z|} \frac{\bar{z}-\bar{\zeta}}{z-\zeta} d \lambda(\zeta)\right|
\end{aligned}
$$

and

$$
\mathrm{III}=\left|\int_{|\zeta| \leq 2|z|} \frac{\bar{\zeta}}{\zeta} d \lambda(\zeta)\right|
$$


Set $\lambda(r)=\lambda(\Delta(0, r))$. Since the terms II and III are not greater than $\lambda(2|z|)$, to take care of them it is enough to realize that $\lambda(r)=o(r)$, which follows from

$$
\lambda(r) \leq r \int_{|\zeta|<r}|\zeta|^{-1} d \lambda(\zeta)
$$

We must now estimate I. Fix $\zeta \neq 0$ and apply Taylor's formula to the function $\frac{\bar{z}-\bar{\zeta}}{z-\zeta}$ around $z=0$ to get

$$
\frac{\bar{z}-\bar{\zeta}}{z-\zeta}=\frac{\bar{\zeta}}{\zeta}+\frac{\bar{\zeta}}{\zeta^{2}} z-\frac{1}{\zeta} \bar{z}+O\left(\frac{|z|^{2}}{|\zeta|^{2}}\right) .
$$

Using the definition of $\alpha$ and $\beta$ we obtain

$$
\begin{aligned}
I \leq & |z|\left|\int_{|\zeta| \leq 2|z|} \overline{\zeta \zeta^{-2}} d \lambda(\zeta)\right|+|z|\left|\int_{|\zeta| \leq 2|z|} \zeta^{-1} d \lambda(\zeta)\right| \\
& +C|z|^{2} \int_{|\zeta|>2|z|}|\zeta|^{-2} d \lambda(\zeta) .
\end{aligned}
$$

The first two terms can be estimated by $|z| \int_{|\zeta| \leq 2|z|}|\zeta|^{-1} d \lambda(\zeta)$ which is $o(|z|)$. To take care of the third it is enough to show that $r \int_{r}^{\infty} d \lambda(t) / t^{2}$ $\rightarrow 0$ as $r \rightarrow 0$. This follows from

$$
\int_{r}^{\infty} \frac{d \lambda(t)}{t^{2}}=-\frac{\lambda(r)}{r^{2}}+\int_{r}^{\infty} \frac{\lambda(t)}{t^{3}} d t
$$

and l'Hôpital's rule, which gives

$$
\lim _{r \rightarrow 0} r \int_{r}^{\infty} \frac{\lambda(t)}{t^{3}} d t=\lim _{r \rightarrow 0} \frac{\lambda(r)}{r}=0 .
$$

Proof of 4.1. Keep the notation in the argument following the statement of 4.1. Let $E$ stand for the set of points at which $F$ vanishes and has a non-zero ordinary differential.

Lemma 2.3 in [7] tells us that $E$ has $\sigma$-finite length and consequently zero newtonian capacity. On the other hand, the hypothesis on $\mu$ guarantees that $\mu$ vanishes on sets of zero newtonian capacity. Therefore $\nabla F=0 \quad \mu$-almost everywhere and so

$$
\begin{aligned}
& \int P(z) d \lambda(z)=-\int \bar{\partial} F(z) d \mu(z)=0, \\
& \int Q(z) d \lambda(z)=\int \partial F(z) d \mu(z)=0 .
\end{aligned}
$$


Since $\bar{\partial}\left(P^{2}\right)=2 \pi P \mu$,

$$
\int P^{2}(z) d \lambda(z)=-2 \int \bar{\partial} F(z) P(z) d \mu(z)=0 .
$$

To complete the proof of 4.1 we are going to show that

$$
\int P Q d \lambda=-\int \bar{\partial} F(z) Q(z) d \mu(z)+\int \partial F(z) P(z) d \mu(z) .
$$

Set $G=\frac{\bar{z}}{z} * \mu$ and consider regularizations $\lambda_{\varepsilon}$ and $F_{\varepsilon}$ of $\lambda$ and $F$. Since $F_{\varepsilon}$ is a smooth compactly supported function, one has

$$
\begin{aligned}
\int P Q d \lambda_{\varepsilon} & =-\frac{1}{\pi} \int \bar{\partial} G \partial G \bar{\partial}^{2} F_{\varepsilon} d x d y \\
& =-\int \bar{\partial} F_{\varepsilon} Q d \mu+\frac{1}{\pi} \int \bar{\partial} F_{\varepsilon}(z) \bar{\partial} G(z) \bar{\partial} \partial G(z) .
\end{aligned}
$$

The last integral above is equal to

$$
\begin{aligned}
\frac{1}{\pi} \int \bar{\partial} F_{\varepsilon} \bar{\partial} G \partial \bar{\partial} G & =\frac{1}{\pi} \int \bar{\partial} F_{\varepsilon} \partial\left(\frac{P^{2}}{2}\right) \\
& =\frac{1}{\pi} \int \partial F_{\varepsilon} \bar{\partial}\left(\frac{P^{2}}{2}\right)=\int \partial F_{\varepsilon} P d \mu,
\end{aligned}
$$

and thus

$$
\int P Q d \lambda_{\varepsilon}=-\int \bar{\partial} F_{\varepsilon} Q d \mu+\int \partial F_{\varepsilon} P d \mu .
$$

To get (8) from (9) by letting $\varepsilon \rightarrow 0$ one only has to apply the Lebesgue dominated convergence theorem to the right-hand side of (9), which is justified by the inequality

$$
\frac{1}{|z|} * d\left|\lambda_{\varepsilon}\right| \leq C \frac{1}{|z|} * d|\lambda|
$$

and the fact that $\mu$ vanishes on sets of zero newtonian capacity.

5. Proof of the theorem. Let $f \in h\left(X, \bar{\partial}^{2}\right)$ be Dini-continuous on $X$. We can extend $f$ to a compactly supported Dini-continuous function on the whole plane $\mathbb{C}[9$, Chapter VI] with modulus of continuity $w(\delta)$. Fix $\delta>0$ and consider a $\delta$-Vitushkin scheme $\left(\Delta_{j}, \varphi_{j}, f_{j}\right)$ for the approximation of $f$. This means that $\left(\Delta_{j}\right)$ is an almost disjoint covering of $\mathbb{C}$ by discs of radius $\delta,\left(\varphi_{j}\right)$ is a partition of the unity subordinated to $\left(\Delta_{j}\right)$ satisfying $\left|\nabla^{l} \varphi_{j}\right| \leq C \delta^{-l}, 0 \leq l \leq 2$, for all $j$, and $f_{j}=\frac{1}{\pi} \frac{\bar{z}}{z} * \varphi_{j} \bar{\partial}^{2} f$. It turns out that $f_{j}$ is continuous on $\mathbb{C}$, 
$f_{j} \in h\left(X, \bar{\partial}^{2}\right),\left\|f_{j}\right\|_{\infty} \leq C w(\delta)$ and $f=\sum_{j} f_{j}$. Each $f_{j}$ has an expansion outside $\Delta_{j}$ of the form (we assume $\Delta_{j}$ is centered at 0 )

$$
f_{j}(z)=c_{0} \frac{\bar{z}}{z}+\frac{b_{1}}{z}+c_{1} \frac{\bar{z}}{z^{2}}+\cdots+\frac{b_{n}}{z^{n}}+c_{n} \frac{\bar{z}}{z^{n+1}}+\cdots,
$$

where $c_{n}=\pi^{-1}\left\langle\bar{\partial}^{2} f_{j}, z^{n}\right\rangle$ and $b_{n}=-\pi^{-1}\left\langle\bar{\partial}^{2} f_{j}, z^{n-1} \bar{z}\right\rangle$, the brackets meaning the duality between compactly supported distributions and $C^{\infty}$ functions. From these formulas one easily shows that $\left|c_{n}\right|$ and $\left|b_{n}\right|$ are estimated by some constant times $\delta^{n} w(\delta)$.

The goal of the next lemma is to find functions $g_{j}$ in $H\left(X, \bar{\partial}^{2}\right)$ whose expansion outside $\Delta_{j}$ coincides with that of $f_{j}$ up to order 2 .

5.1. Lemma. Let $\Delta$ be a disc of center a and radius $\delta$ and $\varphi \in$ $C_{0}^{2}(\Delta)$ with $\left|\nabla^{l} \varphi\right| \leq C \delta^{-l}, 0 \leq l \leq 2$. Set $f_{\Delta}=\frac{1}{\pi} \frac{\bar{z}}{z} * \varphi \bar{\partial}^{2} f$ and let $E$ be the (compact) support of $\bar{\partial}^{2} f_{\Delta}$. Then there exists $g_{\Delta} \in H\left(X, \bar{\partial}^{2}\right)$ such that $\left\|g_{\Delta}\right\|_{\infty} \leq C \varepsilon(\delta)$ and

$$
\left|f_{\Delta}(z)-g_{\Delta}(z)\right| \leq C \varepsilon(\delta) \delta C(E)|z-a|^{-2}, \quad|z-a| \geq 3 \delta,
$$

where $\varepsilon(\delta)$ is defined by (4).

Proof. We apply Lemma 2.2 to the set $E$ and the measure function $h(t)=w(t) t, w$ being the modulus of continuity of $f$. We then get a family of discs $\left\{\Delta_{j}\right\}$ satisfying properties from (a) to (e) in 2.2 and also (f) in Remark 1.

Take now $\varphi_{j} \in C_{0}^{2}\left(\lambda \Delta_{j}\right),\left|\nabla^{l} \varphi_{j}\right| \leq C \delta^{-l}, 0 \leq l \leq 2$, such that $\sum_{j} \varphi_{j}=1$ on $\bigcup_{j} \Delta_{j}$. Set

$$
F_{j}=\frac{1}{\pi} \frac{\bar{z}}{z} * \varphi_{j} \bar{\partial}^{2} f_{\Delta}=\frac{1}{\pi} \frac{\bar{z}}{z} * \varphi_{j} \varphi \bar{\partial}^{2} f,
$$

so that $f_{\Delta}=\sum_{j} F_{j}$ and $\left\|F_{j}\right\|_{\infty} \leq C w\left(\delta_{j}\right)$. Let us call an index $j$ good or bad according to whether the disc $\Delta_{j}$ is good or bad.

If $j$ is a good index, then $\Delta_{j}$ has the three points property with respect to $E \subset \mathbb{C} \backslash \stackrel{\circ}{X}$, and consequently with respect to $\mathbb{C} \backslash X$. Therefore, by Lemma 2.1 we can find $G_{j} \in H\left(X, \bar{\partial}^{2}\right)$ satisfying $\left\|G_{j}\right\|_{\infty} \leq$ $C w\left(\delta_{j}\right)$ and $F_{j}=G_{j}+O\left(|z|^{-2}\right)$ as $z \rightarrow \infty$.

If $j$ is a bad index, then $G_{j} \in H\left(X, \bar{\partial}^{2}\right)$ can be found [7, p. 310] with $\left\|G_{j}\right\|_{\infty} \leq C w\left(\delta_{j}\right)$ and $F_{j}=G_{j}+O\left(|z|^{-1}\right)$ as $z \rightarrow \infty$.

Set $G_{\Delta}=\sum_{j} G_{j}$. Then

$$
\left\|G_{\Delta}\right\|_{\infty} \leq\left\|f_{\Delta}-G_{\Delta}\right\|_{\infty}+C w(\delta)
$$


and

$$
\left\|f_{\Delta}-G_{\Delta}\right\|_{\infty} \leq\left\|\sum_{j \text { bad }} F_{j}-G_{j}\right\|_{\infty}+\left\|\sum_{j \text { good }} F_{j}-G_{j}\right\|_{\infty} \leq C \varepsilon(\delta),
$$

because of Lemma 3.1 and the properties of $\left\{\Delta_{j}\right\}$.

We need now to modify $G_{\Delta}$ to get a $g_{\Delta}$ satisfying (10). Assume that the center of $\Delta$ is the origin and expand $H \equiv f_{\Delta}-G_{\Delta}$ at $\infty$ :

$$
H(z)=\frac{b_{1}}{z}+c_{1} \frac{\bar{z}}{z^{2}}+\cdots+\frac{b_{n}}{z^{n}}+c_{n} \frac{\bar{z}}{z^{n+1}}+\cdots .
$$

To estimate $b_{n}$ and $c_{n}$ we must also consider the expansions of $H_{j} \equiv$ $F_{j}-G_{j}\left(z_{j}\right.$ is the center of $\left.\Delta_{j}\right)$ :

$$
\begin{aligned}
H_{j}(z)= & \frac{b_{1}^{j}}{z-z_{j}}+c_{1}^{j} \frac{\bar{z}-\bar{z}_{j}}{\left(z-z_{j}\right)^{2}}+\cdots+\frac{b_{n}^{j}}{\left(z-z_{j}\right)^{n}} \\
& +c_{n}^{j} \frac{\bar{z}-\bar{z}_{j}}{\left(z-z_{j}\right)^{n+1}}+\cdots
\end{aligned}
$$

Recall that if $j$ is good then $b_{1}^{j}=c_{1}^{j}=0$, so that

$$
\left|b_{1}\right|=\left|\sum_{j \text { bad }} b_{1}^{j}\right| \leq \sum_{j \text { bad }} C w\left(\delta_{j}\right) \delta_{j} \leq C M^{h}(E) \leq C \varepsilon(\delta) C(E),
$$

where the last estimate comes from $\S 2(\mathrm{~A})$ and in the next to the last inequality (d) of 2.2 was used. Similarly one gets $\left|c_{1}\right| \leq C \varepsilon(\delta) C(E)$.

For $n \geq 2, \pi\left|c_{n}\right|$ is equal to

$$
\left|\left\langle\bar{\partial}^{2} H, z^{n}\right\rangle\right| \leq \sum_{j}\left|\left\langle\bar{\partial}^{2} H_{j}, z^{n}\right\rangle\right| .
$$

Writing $z^{n}=\sum_{m=0}^{n}\left(\begin{array}{c}n \\ m\end{array}\right) z_{j}^{n-m}\left(z-z_{j}\right)^{m}$, we can estimate $\left|\left\langle\bar{\partial}^{2} H_{j}, z^{n}\right\rangle\right|$ by

$$
\sum_{m=1}^{n}\left(\begin{array}{c}
n \\
m
\end{array}\right)\left|z_{j}\right|^{n-m}\left|\left\langle\bar{\partial}^{2} H_{j},\left(z-z_{j}\right)^{m}\right\rangle\right|=\pi \sum_{m=1}^{n}\left(\begin{array}{c}
n \\
m
\end{array}\right)\left|z_{j}\right|^{n-m}\left|c_{m}^{j}\right| .
$$

Since $c_{1}^{j}=0$ for good indexes $j$ we have

$$
\left|c_{n}\right| \leq C \delta^{n-1} \sum_{j \text { bad }} w\left(\delta_{j}\right) \delta_{j}+\sum_{m=2}^{n}\left(\begin{array}{c}
n \\
m
\end{array}\right) \delta^{n-m} \sum_{j} w\left(\delta_{j}\right) \delta_{j}^{m} .
$$

Now properties (e) and (f) of the family $\left\{\Delta_{j}\right\}$ give

$$
\left|c_{n}\right| \leq C 2^{n-1} \varepsilon(\delta) \delta^{n-1} C(E) \text {. }
$$


One proves the same estimate for $\left|b_{n}\right|$ arguing similarly.

Let $\mu$ be a positive measure, with support in $E$, such that $\frac{1}{|z|} * \mu$ is continuous on $\mathbb{C}, \frac{1}{|z|} * \mu \leq 1$ and $2\|\mu\| \geq C(E)$. Set

$$
P=\frac{1}{z} * b_{1}(\mu /\|\mu\|)+\frac{\bar{z}}{z^{2}} * c_{1}(\mu /\|\mu\|) \text {. }
$$

Then $\|P\|_{\infty} \leq C \varepsilon(\delta)$ and $P \in H\left(X, \bar{\partial}^{2}\right)$ according to Lemma 4.1.

Define $g_{\Delta}=G_{\Delta}+P$. Then the expansion of $f_{\Delta}-g_{\Delta}=f_{\Delta}-G_{\Delta}-P$ outside $\Delta$ is of the form

$$
f_{\Delta}(z)-g_{\Delta}(z)=\frac{b_{2}^{*}}{z^{2}}+c_{2}^{*} \frac{\bar{z}}{z^{3}}+\cdots+\frac{b_{n}^{*}}{z^{n}}+c_{n}^{*} \frac{\bar{z}}{z^{n+1}}+\cdots
$$

where $b_{n}^{*}=b_{n}-b_{n}(P)$ and $c_{n}^{*}=c_{n}-c_{n}(P), b_{n}(P)$ and $c_{n}(P)$ being the coefficients in the expansion of $P$. We wish now to estimate $\left|b_{n}^{*}\right|$ and $\left|c_{n}^{*}\right|$ by $C 2^{n-1} \varepsilon(\delta) \delta^{n-1} C(E)$, which is easy, because we have even better estimates for $\left|b_{n}(P)\right|$ and $\left|c_{n}(P)\right|$. For example

$$
\left|c_{n}(P)\right|=\left|\int z^{n-1} b_{1}\|\mu\|^{-1} d \mu(z)\right| \leq \delta^{n-1}\left|b_{1}\right| \leq C \delta^{n-1} \varepsilon(\delta) C(E) .
$$

Therefore for $|z|>3 \delta$ we get

$$
\left|f_{\Delta}(z)-g_{\Delta}(z)\right| \leq \sum_{n=2}^{\infty} C 2^{n-1} \varepsilon(\delta) \delta^{n-1} C(E)|z|^{-n} \leq C \varepsilon(\delta) \delta C(E)|z|^{-2},
$$

and this completes the proof of the lemma.

We go back now to the proof of the theorem. We used a $\delta$-Vitushkin scheme to express $f$ as $\sum_{j} f_{j}$. Fix $j$. Let $E$ be the support of $\bar{\partial}^{2} f_{j}$ and let $\gamma=C(E)$. We sublocalize $f_{j}$ according to a $\gamma$-Vitushkin scheme $\left(D_{k}, \psi_{k}, F_{k}\right)$, where $F_{k}=\frac{1}{\pi} \frac{\bar{z}}{z} * \psi_{k} \bar{\partial}^{2} f_{j}=\frac{1}{\pi} \frac{\bar{z}}{z} * \psi_{k} \varphi_{j} \bar{\partial}^{2} f$. Thus $f_{j}=\sum_{k} F_{k}$ and $\left\|F_{k}\right\|_{\infty} \leq c w(\gamma)$. Apply now Lemma 5.1 to $D_{k}$ and $F_{k}$ to get $G_{k} \in H\left(X, \bar{\partial}^{2}\right)$ such that $\left\|G_{k}\right\|_{\infty} \leq C \varepsilon(\gamma)$ and

$$
\left|F_{k}(z)-G_{k}(z)\right| \leq C \varepsilon(\gamma) \gamma C\left(E_{k}\right)\left|z-z_{k}\right|^{-2}, \quad\left|z-z_{k}\right|>3 \gamma,
$$

where $z_{k}$ is the center of $D_{k}$ and $E_{k}$ the support of $\bar{\partial}^{2} F_{k}$.

We would like at this point to apply a variant of a well known lemma of Vitushkin ([3,2.7, p. 202]). We present a proof of the result we need for the reader's convenience and also because the argument gives a quick new proof of Vitushkin's lemma. 
5.2. LEMMA. Let $E$ be a Borel subset of the plane with newtonian capacity $C(E)=\gamma$. Assume that $\left\{\Delta_{k}\right\}$ is an almost disjoint family of discs of radius $\gamma$ and set $E_{k}=E \cap \Delta_{k}$. Then for some constant $C$,

$$
\sum_{k} C\left(E_{k}\right) \leq C C(E)
$$

and

$$
\sum_{\left|z-z_{k}\right|>2 \gamma} \frac{C\left(E_{K}\right)}{\left|z-z_{k}\right|} \leq C, \quad z \in \mathbb{C} .
$$

Proof. By Hölder's inequality with exponents 3 and $3 / 2$

$$
\begin{aligned}
\sum_{\left|z-z_{k}\right|>2 \gamma} \frac{C\left(E_{k}\right)}{\left|z-z_{k}\right|} & \leq\left(\sum_{k}\left(\frac{C\left(E_{k}\right)}{\gamma}\right)^{3 / 2}\right)^{2 / 3}\left(\sum_{\left|z-z_{k}\right|>2 \gamma} \frac{\gamma^{3}}{\left|z-z_{k}\right|^{3}}\right)^{1 / 3} \\
& \leq\left(\sum_{k} \frac{C\left(E_{k}\right)}{C(E)}\right)^{2 / 3}\left(\gamma \int_{|\zeta-z|>\gamma} \frac{d x d y}{|\zeta-z|^{3}}\right)^{1 / 3} \\
& =C\left(\sum_{k} \frac{C\left(E_{k}\right)}{C(E)}\right)^{2 / 3} .
\end{aligned}
$$

Let $\mu_{k}$ be a positive measure with support in $E_{k}, 2\left\|\mu_{k}\right\| \geq C\left(E_{k}\right)$ and $\frac{1}{|z|} * \mu_{k} \leq 1$ on $\mathbb{C}$. For $\left|z-z_{k}\right|>2 \gamma$ we have

$$
\int \frac{d \mu_{k}(\zeta)}{|z-\zeta|} \leq C \frac{C\left(E_{k}\right)}{\left|z-z_{k}\right|}
$$

and hence, setting $\mu=\sum_{k} \mu_{k}$,

$$
\int \frac{d \mu(\zeta)}{|z-\zeta|} \leq C+C\left(\sum_{k} \frac{C\left(E_{k}\right)}{C(E)}\right)^{2 / 3} .
$$

The definition of $C(E)$ now gives

$$
\sum_{k} \frac{C\left(E_{k}\right)}{C(E)} \leq C+C\left(\sum_{k} \frac{C\left(E_{k}\right)}{C(E)}\right)^{2 / 3}
$$

and so

$$
\sum_{k} C\left(E_{k}\right) \leq C C(E),
$$

which completes the proof of the lemma. 
We proceed with the last step in the proof of the theorem. We claim that

$$
\left\|\sum_{k} G_{k}\right\|_{\infty} \leq C \varepsilon(\delta)
$$

Clearly

$$
\left\|\sum_{k} G_{k}\right\|_{\infty} \leq\left\|\sum_{k} F_{k}-G_{k}\right\|_{\infty}+C w(\delta) .
$$

Using the decay estimate (10) and 5.2 we get

$$
\sum_{k}\left|F_{k}(z)-G_{k}(z)\right| \leq C \varepsilon(\gamma)+C \varepsilon(\gamma) \sum_{\left|z-z_{k}\right|>3 \gamma} \frac{C\left(E_{k}\right)}{\left|z-z_{k}\right|} \leq C \varepsilon(\gamma),
$$

which proves the claim.

Set $g_{j}=\sum_{k} G_{k}$ and consider the expansions

$$
\begin{aligned}
& f_{j}(z)-g_{j}(z)=\frac{b_{2}}{z_{2}}+c_{2} \frac{\bar{z}}{z^{3}}+O\left(|z|^{-3}\right) \quad \text { as } z \rightarrow \infty \\
& F_{k}(z)-G_{k}(z)=\frac{b_{2}^{k}}{\left(z-z_{k}\right)^{2}}+c_{2}^{k} \frac{\bar{z}-\bar{z}_{k}}{\left(z-z_{k}\right)^{3}}+O\left(|z|^{-3}\right) \quad \text { as } z \rightarrow \infty .
\end{aligned}
$$

We have by 5.2

$$
\left|b_{2}\right|=\left|\sum_{k} b_{2}^{k}\right| \leq \sum_{k} C \varepsilon(\gamma) \gamma C\left(E_{k}\right) \leq C \varepsilon(\gamma) \gamma C(E) \leq C \varepsilon(\delta) C(E)^{2},
$$

and similarly $\left|c_{2}\right| \leq C \varepsilon(\delta) C(E)^{2}$.

Let $\mu$ be a positive measure, with support in $E$, such that $\frac{1}{|z|} * \mu$ is continuous, $\frac{1}{|z|} * \mu \leq 1$ and $2\|\mu\| \geq C(E)$.

Set $P=(1 / z) *(\mu /\|\mu\|)$ and $Q=\left(\bar{z} / z^{2}\right) *(\mu /\|\mu\|)$. Then

$$
\begin{gathered}
b_{2} P^{2}(z)=\frac{b_{2}}{z^{2}}+O\left(|z|^{-3}\right) \quad \text { as } z \rightarrow \infty, \\
c_{2} P(z) Q(z)=c_{2} \frac{\bar{z}}{z^{3}}+O\left(|z|^{-3}\right) \quad \text { as } z \rightarrow \infty,
\end{gathered}
$$

$\left\|b_{2} P^{2}\right\|_{\infty} \leq C \varepsilon(\delta)$ and $\left\|c_{2} P Q\right\|_{\infty} \leq C \varepsilon(\delta)$.

The function $h_{j}=g_{j}+b_{2} P^{2}+c_{2} P Q$ belongs to $H\left(X, \bar{\partial}^{2}\right)$ because of 4.1. Since 


$$
\begin{gathered}
f_{j}-h_{j}=O\left(|z|^{-3}\right) \quad \text { as } z \rightarrow \infty, \\
\left\|f-\sum_{j} h_{j}\right\|_{\infty}=\left\|\sum_{j} f_{j}-h_{j}\right\|_{\infty} \leq C \max _{j}\left\|f_{j}-h_{j}\right\|_{\infty} \leq C \varepsilon(\delta),
\end{gathered}
$$

and thus $\sum_{j} h_{j}$ is the desired approximant in $H\left(X, \bar{\partial}^{2}\right)$.

6. The string of beads. It is a set of the form $X=\bar{D} \backslash\left(\bigcup_{k} D_{k}\right)$ where $D$ is the open unit disc, the $D_{k}$ are open discs centered on the interval $I=[-1 / 2,1 / 2]$ such that $\bar{D}_{k} \subset D, \bar{D}_{k} \cap \bar{D}_{l}=\varnothing$ if $k \neq l$ and $\bigcup D_{k}$ is dense in $I$.

We wish to prove that (1) holds for $X$. Take $f \in h\left(X, \bar{\partial}^{2}\right)$ and write $f=\sum_{j} f_{j}$ using a $\delta$-Vitushkin scheme $\left(\Delta_{j}, \varphi_{j}, f_{j}\right)$. If $\Delta_{j}$ does not intersect $I$ then $f_{j} \in H\left(X, \bar{\partial}^{2}\right)$ (by [2, Theorem 2]). If $\Delta_{j} \cap I \neq \varnothing$, replacing $\Delta_{j}$ by a disc of comparable size, we can assume that the center $c$ of $\Delta_{j}$ lies in $I$. Using two points in $\Delta_{j} \cap\left(\bigcup_{k} D_{k}\right)$ at distance not less than $\delta / 2$, we find, by a variant of 2.1 , a function $g_{j} \in H\left(X, \bar{\partial}^{2}\right)$ such that $\left\|f_{j}\right\|_{\infty} \leq C w(\delta)$ and $f_{j}(z)-g_{j}(z)=$ $b /(z-c)+O\left(|z|^{-2}\right)$ as $z \rightarrow \infty$.

We claim now that

$$
|b| \leq C w(\delta) \alpha\left(\Delta_{j} \backslash X\right) .
$$

Once this is proved we consider $h_{j}$ continuous on $\mathbb{C}$, analytic off a compact subset of $\Delta_{j} \backslash X,\left\|h_{j}\right\|_{\infty} \leq C w(\delta)$ and $h_{j}(z)=b /(z-c)+$ $O\left(|z|^{-2}\right)$ as $z \rightarrow \infty$. Then $K_{j}=g_{j}+h_{j} \in H\left(X, \bar{\partial}^{2}\right),\left\|K_{j}\right\|_{\infty} \leq$ $C w(\delta)$ and $f_{j}-K_{j}=O\left(|z|^{-2}\right)$ as $z \rightarrow \infty$.

Consequently

$$
\left\|\sum_{\Delta, \cap I \neq \varnothing} f_{j}-K_{j}\right\|_{\infty} \leq C w(\delta),
$$

and so $\sum_{\Delta_{j} \cap I=\varnothing} f_{j}+\sum_{\Delta_{J} \cap I \neq \varnothing} K_{j}$ is the desired approximant in $H\left(X, \bar{\partial}^{2}\right)$.

To prove (11), given $\varepsilon>0$, we cover $\left(\Delta_{j} \cap I\right) \backslash\left(\bigcup_{k} D_{k}\right)$ by discs $B_{l}$ of radius $r_{l}=\varepsilon$ such that $\sum_{l} r_{l} \leq 2 \delta$. It is not difficult to realize that $\bigcup D_{k}$ can be covered by a family of discs $D_{m}^{*}$ of radii $r_{m}^{*} \leq \delta$ such that $\sum r_{m}^{*} \leq C$ length $\left(\left(\Delta_{j} \backslash X\right) \cap I\right)$. Write now $\left\{B_{l}\right\} \cup\left\{D_{m}^{*}\right\}=\left\{D_{n}^{* *}\right\}$ and consider a partition of the unity $\left(\varphi_{n}\right)$ subordinated to the above covering and satisfying $\left|\nabla^{i} \varphi_{n}\right| \leq C \delta^{-i}, 0 \leq i \leq 2$. Set $d_{j}=f_{j}-g_{j}$. 
Denoting by $z_{n}$ the center of $D_{n}^{* *}$ we then have

$$
\begin{aligned}
\pi|b| & =\left|\left\langle\bar{\partial}^{2} d_{j}, \bar{z}\right\rangle\right|=\left|\left\langle\bar{\partial}^{2} d_{j}, \bar{z}-z\right\rangle\right| \\
& \leq 2 \sum_{n}\left|\left\langle\bar{\partial}^{2} d_{j}, \varphi_{n}(z) \operatorname{Im} z\right\rangle\right| \\
& \leq 2 \sum_{n} \int\left|d_{j}(z)-d_{j}\left(z_{n}\right)\right|\left|\operatorname{Im} z \bar{\partial}^{2} \varphi_{n}(z)+\bar{\partial} \varphi_{n}(z)\right| d x d y \\
& \leq C \sum_{l} w(\varepsilon) r_{l}+C \sum_{m} w(\delta) r_{m}^{*} \\
& \leq C w(\varepsilon) \delta+C w(\delta) \text { length }\left(\left(\Delta_{j} \backslash X\right) \cap I\right) .
\end{aligned}
$$

Letting $\varepsilon \rightarrow 0$ we get

$$
|b| \leq C w(\delta) \gamma\left(\left(\Delta_{j} \backslash X\right) \cap I\right) \leq C w(\delta) \gamma\left(\Delta_{j} \backslash X\right)=C w(\delta) \alpha\left(\Delta_{j} \backslash X\right),
$$

where we used that four times the analytic capacity $\gamma$ of a subset of the line is equal to its length and that $\gamma$ and $\alpha$ coincide on open subsets of the plane.

Acknowledgments. The author is extremely grateful for the generous support given at different stages by the following institutions: CIRIT (Generalitat de Catalunya), DGPC (Mec) and DGICYT (PB89-0311).

I am also indebted to the Mathematics Departments of the Maynooth College and UCLA for their warm hospitality.

\section{REFERENCES}

[1] J. J. Carmona, Mergelyan's approximation theorem for rational modules, J. Approx. Theory, 44 (1985), 113-125.

[2] J. Deny, Systèmes totaux de fonctions harmoniques, Ann. Inst. Fourier, 1 (1949), 103-113.

[3] T. W. Gamelin, Uniform Algebras, Prentice-Hall, Englewood Cliffs, N.J., 1969.

[4] J. Garnett, Analytic Capacity and Measure, Lecture Notes in Math., vol 297, Springer-Verlag, Berlin and New York, 1972.

[5] L. I. Hedberg, Two approximation problems in functions spaces, Ark. Mat., 16 (1978), 51-81.

[6] M. V. Keldysh, On the solubility and stability of the Dirichlet problem, Amer. Math. Soc. Transl., (2) 51 (1966), 1-73.

[7] J. Mateu and J. Verdera, BMO harmonic approximation in the plane and spectral synthesis for Hardy-Sobolev spaces, Revista Matem. Iberoamericana, 4 (1988), 291-318.

[8] M. Melnikov and J. Orobitg, A counterexample to a Vitali type theorem for planar sets and one dimensional Hausdorff content, to appear in Proc. Amer. Math. Soc.

[9] E. M. Stein, Singular integrals and differentiability properties of functions, Princeton Univ. Press, Princeton, N.J., 1970. 
[10] T. Trent and J. L. Wang, Uniform approximation by rational modules on nowhere dense sets, Proc. Amer. Math. Soc., 81 (1981), 62-64.

[11] A. G. Vitushkin, Analytic capacity of sets in problems of approximation theory, Russian Math. Surveys, 22 (1967), 139-200.

[12] J. L. Wang, A localization operator for rational modules, Rocky Mountain J. Math., 19 (1989), 999-1002.

Received August 5, 1991 and in revised form March 16, 1992.

Universitat Autonoma de Barcelona

08193 BELLATERRA (BARCELONA)

SPAIN 


\title{
PACIFIC JOURNAL OF MATHEMATICS \\ Founded by \\ E. F. BecKenbaCH (1906-1982) F. Wolf (1904-1989)
}

\section{EDITORS}

V. S. VARADARAJAN
(Managing Editor)
University of California
Los Angeles, CA 90024-1555
vsv@math.ucla.edu
F. MICHAEL CHRIsT
University of California
Los Angeles, CA 90024-1555
christ@math.ucla.edu
HerBeRT CLEMENs
University of Utah
Salt Lake City, UT 84112
clemens@math.utah.edu

V. S. VARADARAJAN University of California Los Angeles, CA 90024-1555 @math.ucla.edu Los Angeles, CA 90024-1555

Herbert Clemens clemens@math.utah.edu

\author{
THOMAS ENRIGHT \\ University of California, San Diego \\ La Jolla, CA 92093 \\ tenright@ucsd.edu \\ Nicholas ERcolani \\ University of Arizona \\ Tucson, AZ 85721 \\ ercolani@math.arizona.edu \\ R. FINN \\ Stanford University \\ Stanford, CA 94305 \\ finn@gauss.stanford.edu \\ VAUGHAN F. R. JoNES \\ University of California \\ Berkeley, CA 94720 \\ vfr@math.berkeley.edu
}

\author{
STEVEN KeRCKHOFF \\ Stanford University \\ Stanford, CA 94305 \\ spk@gauss.stanford.edu \\ MarTin SCHARLEMANN \\ University of California \\ Santa Barbara, CA 93106 \\ mgscharl@henri.ucsb.edu \\ HAROld STARK \\ University of California, San Diego \\ La Jolla, CA 92093
}

\section{SUPPORTING INSTITUTIONS}

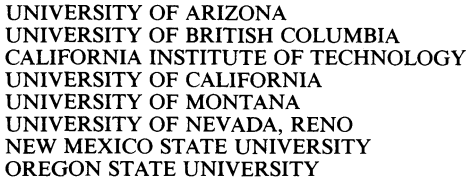

The Supporting Institutions listed above contribute to the cost of publication of this Journal, but they are not owners or publishers and have no responsibility for its content or policies.

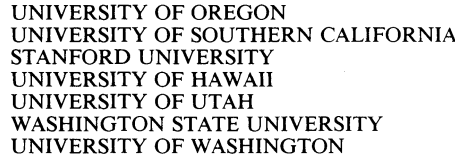

\begin{abstract}
Mathematical papers intended for publication in the Pacific Journal of Mathematics should be in typed form or offset-reproduced (not dittoed), double spaced with large margins. Please do not use built up fractions in the text of the manuscript. However, you may use them in the displayed equations. Underline Greek letters in red, German in green, and script in blue. The first paragraph must be capable of being used separately as a synopsis of the entire paper. In particular it should contain no bibliographic references. Please propose a heading for the odd numbered pages of less than 35 characters. Manuscripts, in triplicate, may be sent to any one of the editors. Please classify according to the 1991 Mathematics Subject Classification scheme which can be found in the December index volumes of Mathematical Reviews. Supply name and address of author to whom proofs should be sent. All other communications should be addressed to the managing editor, or Julie Speckart, University of California, Los Angeles, California 90024-1555.

There are page-charges associated with articles appearing in the Pacific Journal of Mathematics. These charges are expected to be paid by the author's University, Government Agency or Company. If the author or authors do not have access to such Institutional support these charges are waived. Single authors will receive 75 free reprints; joint authors will receive a total of 100 free reprints. Additional copies may be obtained at cost in multiples of 50 .
\end{abstract}

The Pacific Journal of Mathematics (ISSN 0030-8730) is published monthly except for July and August. Regular subscription rate: $\$ 200.00$ a year (10 issues). Special rate: $\$ 100.00$ a year to individual members of supporting institutions.

Subscriptions, orders for numbers issued in the last three calendar years, and changes of address should be sent to Pacific Journal of Mathematics, P.O. Box 969, Carmel Valley, CA 93924, U.S.A. Old back numbers obtainable from Kraus Periodicals Co., Route 100, Millwood, NY 10546.

The Pacific Journal of Mathematics at P.O. Box 969, Carmel Valley, CA 93924 (ISSN 0030-8730) is published monthly except for July and August. Second-class postage paid at Carmel Valley, Califorñia 93924, and additional mailing offices. Postmaster: send address changes to Pacific Journal of Mathematics, P.O. Box 969, Carmel Valley, CA 93924.

\section{PUBLISHED BY PACIFIC JOURNAL OF MATHEMATICS, A NON-PROFIT CORPORATION}

This publication was typeset using $\mathcal{A} \mathcal{M S}-\mathrm{T}_{\mathrm{E}} \mathrm{X}$,

the American Mathematical Society's $T_{E} X$ macro system.

Copyright (C) 1993 by Pacific Journal of Mathematics 


\section{PACIFIC JOURNAL OF MATHEMATICS}

Volume $159 \quad$ No. $2 \quad$ June 1993

$L^{p}$-integrability of the second order derivatives of Green potentials in 201 convex domains

VilHeLM AdOLFSSON

Solutions of the stationary and nonstationary Navier-Stokes equations 227 in exterior domains

ZHi MiN CHEN

Mesures de Patterson-Sullivan sur le bord d'un espace hyperbolique au 241 sens de Gromov

Michel COORNAERT

Differential-difference operators and monodromy representations of 271 Hecke algebras

CHARLES F. DUNKL

Between the unitary and similarity orbits of normal operators

PAUl GuinAND and LAURENT WALSh MARCOUX

Skeins and handlebodies

W. B. RAYMOND LICKORISH

The Plancherel formula for homogeneous spaces with polynomial spectrum

RONALD LESLIE LIPSMAN

On the uniform approximation problem for the square of the

Cauchy-Riemann operator

JoAn Manuel Verdera Melenchón 